\title{
Neurobehavioral effects of treatment for opiate withdrawal
}

\author{
M G Coyle, A Ferguson, L LaGasse, J Liu, B Lester
}

Arch Dis Child Fetal Neonatal Ed 2005;90:F73-F74. doi: 10.1136/adc.2003.046276

\begin{abstract}
A partially randomised, controlled trial was performed to test the hypothesis that opiate exposed infants treated with diluted tincture of opium (DTO) and phenobarbital would have better neurobehavioral scores than infants treated with DTO alone. Compared with those treated with DTO alone (n $=15)$, infants treated with DTO and phenobarbital $(n=17)$ were more interactive, had smoother movements, were easier to handle, and less stressed. Dual treatment results in improved neurobehavioral organisation during the first three weeks of life, which may indicate a more rapid recovery from opiate withdrawal.
\end{abstract}

$\mathrm{T}$ here are currently 250 000-300 000 female intravenous drug abusers in the United States, $75-90 \%$ of whom are child bearing age. ${ }^{1}$ Infants exposed to methadone in utero have a $60-80 \%$ risk of developing neonatal withdrawal. ${ }^{2}$ Studies of the effects of maternal opiate use on the neurobehavioral status of the newborn are outdated and thus do not adequately address contemporary methadone dosing strategies. ${ }^{3}$ The NICU Network neurobehavioral scale (NNNS) was developed for the NIH as part of the maternal lifestyle study of the effects of prenatal drug exposure on child outcome. ${ }^{4}$ This scale was developed as a sensitive tool for assessing drug exposed and other high risk term and preterm infants of 32-44 weeks gestational age.

We have shown previously that the use of diluted tincture of opium (DTO) and phenobarbital for the treatment of opiate withdrawal compared with DTO alone lessens severity of withdrawal, shortens hospital stay by $50 \%$, and reduces hospital cost. ${ }^{5}$ The purpose of this study is to determine the effects of DTO and phenobarbital compared with DTO alone on infant neurobehaviour during the first three weeks of life.

\section{METHODS}

This study was approved by the institutional review boards of Women \& Infants' Hospital of Rhode Island and St Luke's Hospital, New Bedford, Massachusetts, and informed consent was obtained from the mother.

\section{Subject enrolment}

All infants born at St Luke's Hospital between March 1998 and May 2000 to mothers with a history of heroin or methadone use during pregnancy were invited to participate.

\section{Study protocol}

All aspects of care were provided to the infants in the standard fashion in the level II nursery at St Luke's Hospital. This was a partially randomised, controlled trial in which infants were prospectively matched for severity of withdrawal as measured by the first Finnegan score $>7$. Specifically, every subject from the DTO only group had a counterpart from the DTO and phenobarbital group with the same Finnegan score \pm 1 . If the infant had no match, he/she was randomly assigned to a group. Details of the dosing protocol have been published. ${ }^{5}$

The NNNS was administered weekly for three weeks by a certified examiner (AF) masked to the treatment group. The NNNS has been used previously in studies of drug exposed infants. ${ }^{4}$

\section{Calculations and statistical analysis}

One way analysis of variance was used to compare differences between the groups on the sample characteristics. General linear model (GLM, using SPSS 9.0) multivariate analysis for repeated measures was conducted to compare group differences on standard NNNS summary scores across the first three weeks.

\section{RESULTS}

Thirty six infants met entry criteria. Three did not require medication, and one was excluded because of congenital heart disease. The remaining 32 infants received either DTO and placebo $(n=15)$ or DTO and phenobarbital $(n=17)$.

There were no significant differences in the gestational age, birth weight, head circumference, length, maternal methadone dose, or use of illicit substances, alcohol, or cigarettes between the placebo and phenobarbital groups (data not shown, but available on request).

Infants treated with DTO alone remained in hospital for a significantly longer period of time (69.8 (31) v 39.6 (25) days, $\mathrm{p}<0.01)$ and required a greater maximum daily DTO dose (14.8 (12.9) $v 4.3$ (3.0) ml, $\mathrm{p}<0.01$ ) than infants treated with DTO and phenobarbital. These values did not differ significantly when the median values were compared.

As shown in fig 1 , the DTO and phenobarbital group showed improved orientation (Wilks' $\lambda=0.47, \mathrm{p}<0.05$ ) and quality of movement (Wilks' $\lambda=0.53, \mathrm{p}<0.05$ ) during the first three weeks after birth. As such, they were more interactive with their environment and had less resistance to movement. In addition, the DTO and phenobarbital group showed a lower total stress/abstinence score (Wilks' $\lambda=$ $0.66, \mathrm{p}<0.05$, data not shown). Because the total stress differed between the groups, we compared the subscales to determine which organ systems were affected. DTO and phenobarbital infants developed fewer skin stress signs (Wilks' $\lambda=0.52, \mathrm{p}<0.05$ ), such as pallor or mottling, and fewer state stress signs (Wilks' $\lambda=0.62, \mathrm{p}<0.05$ ), such as a high pitched cry or abrupt changes in state (fig 1).

\section{DISCUSSION}

There is no standard treatment regimen for neonatal opiate withdrawal. Furthermore, the neurobehavioral status of the newborn exposed to maternal opiates has not been effectively evaluated using contemporary maternal dosing strategies. The NNNS is a tool designed to be sensitive to drug effects and prematurity.

Abbreviations: DTO, diluted tincture of opium; NNNS, NICU Network neurobehavioral scale 

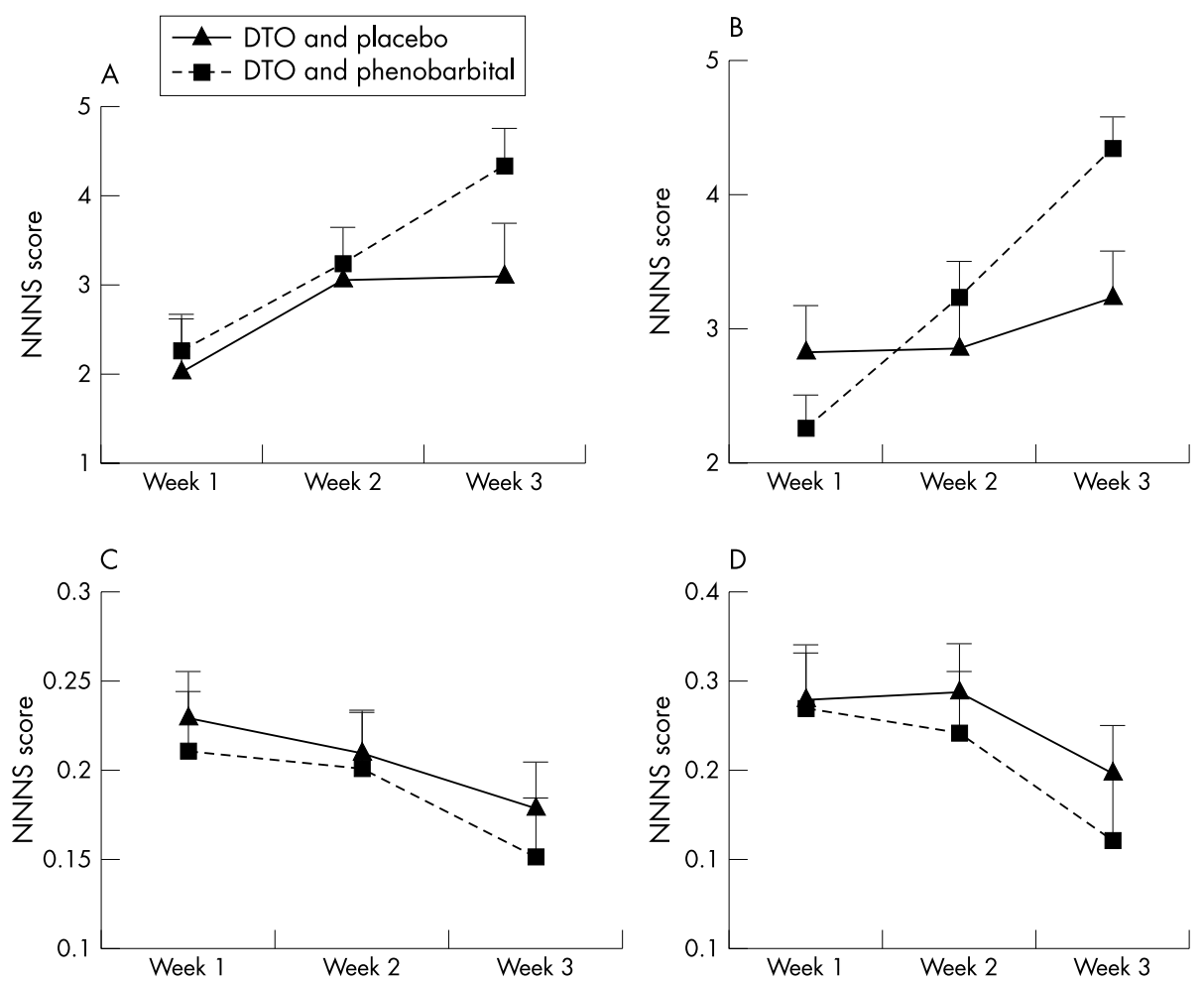

Figure 1 NICU Network neurobehavioral scale (NNNS) scores over time for a group of infants receiving diluted tincture of opium (DTO) and placebo and a group receiving DTO and phenobarbital. Summary scores are given for (A) orientation, (B) quality of movement, (C) skin stress, and (D) state stress. Values are mean (SEM). For orientation and quality of movement, higher scores indicate better performance. The skin and state stress scales indicate the number of stress signs, therefore higher scores indicate worse performance. There was significant $(p<0.05)$ difference between the groups for orientation, quality of movement, skin stress, and state stress.

To our knowledge, this is the first study to show differences in neurobehaviour for different treatment regimens in infants exposed to relatively high maternal methadone doses (mean doses 103 (50) mg and 85.9 (49) mg for DTO alone and DTO and phenobarbital groups). Previous studies used healthy controls for a comparison group to show the neurodevelopmental deficits seen in this at risk population. ${ }^{6}$

Our study shows that, compared with infants treated with DTO alone, those treated with two drugs exhibit improved orientation and quality of movement and fewer stress signs during the first three weeks after birth. Although both groups improved over time, infants receiving the dual therapy recovered more rapidly with evidence of improved neurobehaviour (fig 1).

We did not adjust for multiple comparisons, which would protect against rejecting the null hypothesis when it is correct (type I error). However, the cost of this protection is to erroneously increase the type II error that findings are attributable to chance. ${ }^{7}$ Limiting type II error or maximising sensitivity to find effects is especially critical in this study in which effects are subtle and could easily be missed. We found reliable but small differences attributable to a treatment strategy that are not necessarily deficits.

This is a short term outcome study to compare neurobehavioral differences between two treatment groups after three weeks. Reducing hospital stay and severity of withdrawal not only decreases morbidity, but lessens the societal burden of this growing population. To what extent additional morbidity might develop with the use of short term phenobarbital could not be addressed with this investigation, but would be important to document as part of a long term outcome study. If abnormal infant neurobehaviour is a marker for long term morbidity, the ability to optimise treatment for opiate withdrawal may place these children at decreased risk for long term deficits. Given these early findings in this vulnerable population, a larger study addressing long term outcome should be the focus of future investigations.

\section{Authors' affiliations}

M G Coyle, L LaGasse, J Liu, B Lester, Department of Pediatrics, Brown Medical School, Providence, Rhode Island, USA

A Ferguson, St Luke's Hospital, New Bedford, Massachusetts, USA

Competing interests: none declared

Correspondence to: Dr Coyle, Department of Pediatrics, Brown Medical School, Women \& Infants Hospital, 101 Dudley Street, Providence, Rhode Island 02905-2401, USA; coylem@southcoast.org

Accepted 26 August 2004

\section{REFERENCES}

1 Chang G, Caroll KM, Behr HM, et al. Improving treatment outcome in pregnant opiate-dependent women. J Subst Abuse Treat 1992;9:327-30.

2 Chiriboga CA. Fetal effects. Neurologic complications of drug and alcohol abuse. Neurol Clin 1993;11:707-29.

3 Van Baar L, Fleury P, Soepatmi S, et al. Neonatal behaviour after drug dependent pregnancy. Arch Dis Child 1989;64:235-40.

4 Lester BM, Tronick EZ. Behavioral assessment: The NICU Network neurobehavioral scale. Pediatrics Mar, 2004;113(suppl).

5 Coyle MG, Ferguson A, Lagasse L, et al. Diluted tincture of opium (DTO) and phenobarbital vs. DTO alone for the treatment of neonatal opiate withdrawal in term infants. J Pediatr 2002;140:561-4.

6 Eyler FD, Behnke M. Early development of infants exposed to drugs prenatally. Prenatal drug exposure and child outcome. Clin Perinatol 1999;26:107-50.

7 Rothman KJ. No adjustments are needed for multiple comparisons.

Epidemiology 1990;1:43-6. 